\title{
Perceptual impairment and psychomotor control in virtual laparoscopic surgery
}

\author{
Mark R. Wilson • John S. McGrath · \\ Samuel J. Vine · James Brewer $\cdot$ David Defriend • \\ Richard S. W. Masters
}

Received: 17 June 2010/Accepted: 19 November 2010/Published online: 27 February 2011

(C) The Author(s) 2011. This article is published with open access at Springerlink.com

\begin{abstract}
Background It is recognised that one of the major difficulties in performing laparoscopic surgery is the translation of twodimensional video image information to a three-dimensional working area. However, research has tended to ignore the gaze and eye-hand coordination strategies employed by laparoscopic surgeons as they attempt to overcome these perceptual constraints. This study sought to examine if measures related to tool movements, gaze strategy, and eye-hand coordination (the quiet eye) differentiate between experienced and novice operators performing a two-handed manoeuvres task on a virtual reality laparoscopic surgical simulator (LAP Mentor ${ }^{\mathrm{TM}}$ ).

Methods Twenty-five right-handed surgeons were categorised as being either experienced (having led more than 60 laparoscopic procedures) or novice (having performed fewer than 10 procedures) operators. The 10 experienced and 15 novice surgeons completed the "two-hand manoeuvres" task from the LAP Mentor basic skills learning environment while wearing a gaze registration system. Performance,
\end{abstract}

M. R. Wilson $(\bowtie) \cdot$ S. J. Vine

School of Sport and Health Sciences, University of Exeter,

St Luke's Campus, Exeter EX1 2LU, UK

e-mail: Mark.Wilson@exeter.ac.uk

\section{J. S. McGrath}

Exeter Surgical Health Services Research Unit,

Royal Devon and Exeter NHS Foundation Trust,

Exeter EX2 5DW, UK

J. Brewer · D. Defriend

Centre for Innovation and Training in Elective Care,

Torbay Hospital, Torquay, Devon TQ2 7AA, UK

R. S. W. Masters

Institute of Human Performance, University of Hong Kong,

Pokfulam, Hong Kong movement, gaze, and eye-hand coordination parameters were recorded and compared between groups.

Results The experienced surgeons completed the task significantly more quickly than the novices, used significantly fewer movements, and displayed shorter tool paths. Gaze analyses revealed that experienced surgeons spent significantly more time fixating the target locations than novices, who split their time between focusing on the targets and tracking the tools. A more detailed analysis of a difficult subcomponent of the task revealed that experienced operators used a significantly longer aiming fixation (the quiet eye period) to guide precision grasping movements and hence needed fewer grasp attempts.

Conclusion The findings of the study provide further support for the utility of examining strategic gaze behaviour and eye-hand coordination measures to help further our understanding of how experienced surgeons attempt to overcome the perceptual difficulties inherent in the laparoscopic environment.

Keywords Psychomotor control · Eye-hand coordination · Quiet eye · Gaze strategy · Perception

Due to concerns over patient safety, training via simulation is proposed as a way of supplementing operating theatre experiential learning, especially early in the learning curve $[1,2]$. Recent systematic reviews have demonstrated that such training can translate to improved outcomes in the operating theatre $[3,4]$, although the structure, mode, and design of such training is currently unsystematic $[1,2,5-8]$. The design of training curricula demonstrating validity evidence is therefore a priority for laparoscopic surgery. Such curricula should provide a structure by which an individual can acquire skills to a predetermined level of proficiency, before progressing to more challenging tasks [2]. 
The measurement and the assessment of technical skills are fundamental to the development of a proficiency-led curriculum. Virtual reality (VR) simulators allow such skills to be practiced in a safe, nonthreatening environment. Furthermore, objective performance assessment (time taken, number of errors, and path length for each hand) is provided immediately, and without the need for monitored supervision [9]. Recent studies have provided validity evidence of these measures, with experienced performers tending to be faster, more accurate, and more efficient in their movement paths than their less experienced counterparts [2, 10-14].

However, while one of the primary difficulties in performing laparoscopic surgery is the translation of twodimensional video image information to a three-dimensional working area $[15,16]$, there has been little attention applied to the eye movements and gaze strategies of laparoscopic surgeons [17]. Indeed, the process measures provided by the simulator software reflect the "surgeon-tool" $(\mathrm{S}-\mathrm{T})$ interface (tool movement metrics) as opposed to the "surgeon-monitor" (S-M) interface (eye movement / gaze metrics) [18]. A consideration of gaze metrics may provide an important insight into how operators develop the novel spatial relationships required to overcome the perceptual constraints inherent in the laparoscopic environment. Indeed, while tool movement information might provide a proxy indication of the effect of such perceptual disruptions, explicit measures of gaze control provide direct insights into these effects $[17,19]$.

The aim of the current study was therefore to further our understanding of the psychomotor coordination underlying performance in VR laparoscopic tasks. In addition to comparing expert-novice differences in measures from the separate $\mathrm{S}-\mathrm{T}$ and S-M interfaces (as did Wilson et al. [19]), a more fine-grained analysis of the spatial and temporal coordination between eye and hand movements, the "quiet eye" [20], was performed for a difficult subcomponent of the task. It was hoped that by examining a measure related to eye-hand coordination, as opposed to hand and eye movements separately, greater insight might be gained into how experienced and novice operators attempt to deal with the perceptual constraints imposed by the laparoscopic environment. In other words, can differences in the way in which eye and hand movements are coordinated to overcome the perceptual constraints in the laparoscopic environment differentiate between experienced and novice laparoscopic operators?

\section{Methods}

\section{Participants}

A total of 25 surgeons volunteered to take part in the study (mean age $=32.0$ years; $\quad$ range $=24-49$ years $). \quad$ All participants were right-hand dominant and were classified as novice or experienced laparoscopic surgeons according to the number of laparoscopic procedures they had led. Fifteen novices ( 6 males, 9 females) had performed fewer than 10 procedures and ten experienced operators ( 9 males, 1 female) had led more than 60 procedures (range $=60-700$ ). Power calculations using mean and standard deviations from previous studies using similar tasks and groupings [2, 19] suggest that groups should consist of at least eight individuals for a one-tailed test with $\alpha=0.05$ and power $(1-\beta)=0.8$.

Apparatus and task

Testing took place on a LAP Mentor ${ }^{\mathrm{TM}}$ (Simbionix USA Corp., Cleveland, OH) VR laparoscopic surgical simulator, based at the Centre for Innovation and Training in Elective Care, Torbay Hospital. The two-handed manoeuvres task from the basic skills training module was used for this study as recent research has suggested that it discriminates between levels of expertise across a range of objective performance and S-T measures [2]. To complete the task the operator must locate balls within a jelly mass and then place them in an endobag. There are three subcomponents to this task requiring accurate psychomotor control: (1) grasping the jelly and manipulating it to expose a ball, (2) grasping a ball, and (3) placing the ball in the endobag (Fig. 1).

Participants were fitted with an Applied Science Laboratories Mobile Eye gaze registration system (ASL, Bedford, MA), which measures eye-line of gaze using dark pupil tracking [19]. The system incorporates a pair of lightweight glasses fitted with eye and scene cameras and a set of three LEDs that project harmless near infrared (IR) light onto the eye via a reflective monocle (Fig. 2A). Some of this light is reflected by the cornea (corneal reflection) and appears to the eye camera as a triangle of three dots at a fixed distance from each other. The pupil appears black as light does not exit the inside of the eye, enabling the system to register its position and determine its centre. When the eye turns, the centre of the pupil moves relative to the head, however, the corneal reflection remains in the same position. Therefore, by comparing the vector (angle and distance) between the pupil and the cornea, the eye tracking system can compute the angle at which the eye is pointed (Fig. 2B).

The system also incorporates a recording device (a modified digital video cassette recorder), which combines the two video streams from the eye and scene cameras at $25 \mathrm{~Hz}$. The recorder is attached to a laptop installed with Eyevision (ASL) software; both recorder and laptop were placed on a table to the side of the participant. By teaching the system how the angles calculated by the eye camera 

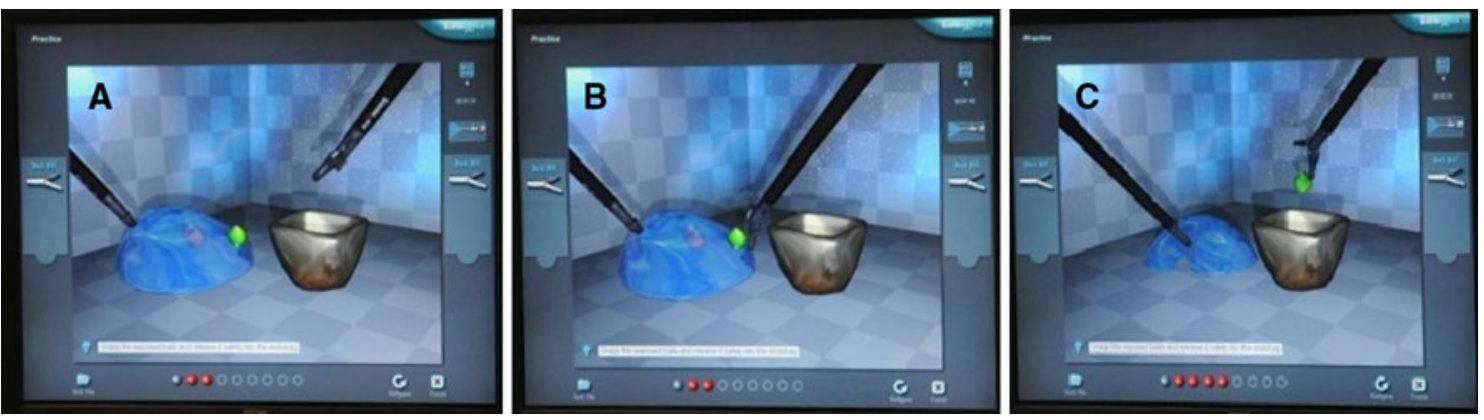

Fig. 1 Images of the LAP Mentor ${ }^{\mathrm{TM}}$ environment showing representative examples of the "two-handed manoeuvres" task: grasping and manipulating the jelly to reveal a ball $(\mathbf{A})$, grasping a ball $(\mathbf{B})$, and dropping a ball in the endobag $(\mathbf{C})$

Fig. 2 The head-mounted unit from the ASL Mobile Eye gaze registration system $(\mathbf{A})$ and the software environment

(B) showing the pupil, the corneal reflection, and the vector line between the two
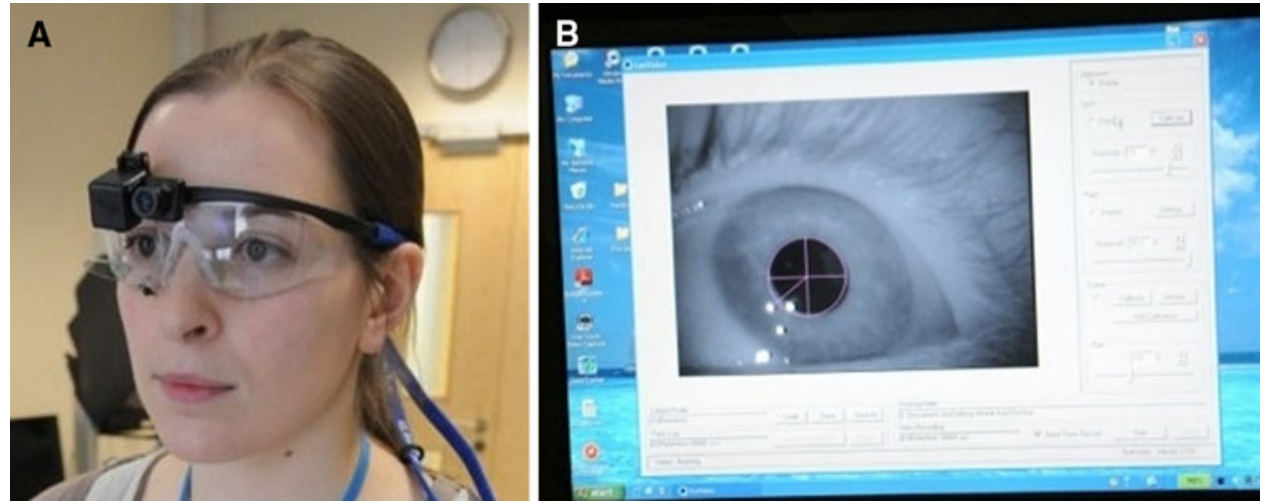

relate to the image from the second camera that is viewing the environment (the scene camera), the eye tracker can compute what the eye is pointed at. A circular cursor, representing $1^{\circ}$ of visual angle with a $4.5-\mathrm{mm}$ lens, indicating the location of gaze in a video image of the scene (spatial accuracy of $\pm 0.5^{\circ}$ visual angle; $0.1^{\circ}$ precision), is viewed in real time and recorded for subsequent offline analyses.

\section{Procedure}

Participants arrived at the Training Centre individually at prearranged times. They first read an information sheet describing the aims of the study before completing a demographic questionnaire and providing written informed consent of participation. Participants were fitted with the eye tracker and it was calibrated using six visual landmarks on the LAP Mentor display screen. They then performed three consecutive attempts at the two-handed manoeuvres task, as part of a series of activities, before being debriefed and thanked for their participation in the study.

\section{Measures}

\section{Complete task}

Task performance was assessed in terms of speed (task completion time), and total path length and number of tool movements were chosen from the $\mathrm{S}-\mathrm{T}$ interface to replicate Aggarwal et al. [2]. Complete task performance and tool process measures were downloaded directly from the LAP Mentor software environment after each trial. The software provided path length and number of movement data for left and right tools and these were aggregated to provide a composite score.

The percentage of time spent fixating on important locations was calculated to provide an overall measure of how the participants attended to the two-dimensional environment (S-M interface) (as in Wilson et al. [19]). The target of interest changes depending on the particular subcomponent of the task being undertaken, i.e., the jelly to be moved in phase 1 , the ball to be lifted in phase 2 , and the endobag in which to place the ball in phase 3 (Fig. 1). For simpler "pointing" tasks in laparoscopic environments, it has been demonstrated that novices tend to switch gaze between tool and target locations in order to determine the relative position of both, whereas experts adopt a more efficient strategy, fixating almost exclusively on the target location [19, 21]. We were interested in determining if similar gaze strategies were adopted for this grasping task which places greater demands on depth perception than pointing.

The gaze data were analysed in a frame-by-frame manner using GazeTracker (Eye Response Technologies, Charlottesville, VA, USA) video analysis software. A 
fixation was defined as a gaze of long enough duration to allow information processing ( $\geq 120 \mathrm{~ms}$ ) to a single location (within $1^{\circ}$ visual angle). For each subcomponent of the task, areas of interest ("lookzones") were created around the relevant subtask target (jelly mould, ball, or endobag) and each tool. These were maintained in place by the experimenter as the video progressed at $25 \mathrm{~Hz}$ (40 frames a second). The software then provided information regarding the duration and frequency of gazes occurring within each area of interest for the duration of the trial.

\section{Subcomponent task: grasping a ball}

In order to provide a more fine-grained analysis of the effects of perceptual constraints on psychomotor control, each attempt at grasping a ball (phase 2) was investigated via video footage analyses. This additional level of analysis sought to answer calls for research examining simulator validity evidence to include outcome measures based on decisive actions during procedures [7]. Ball grasp attempts were selected as they were most sensitive to altered depth perception effects due to the increased degree of accuracy required; the ball diameter is considerably less than either the jelly mould or endobag aperture diameter. The number of attempts required to successfully grasp a ball was defined as the performance measure for this subcomponent, and the quiet eye $(\mathrm{QE})$ was adopted as a specific measure to reflect the spatial and temporal coordination between gaze and motor control [20, 22].

The QE has been shown to underlie higher levels of skill and performance in a wide range of aiming and interceptive skills (see [22] for a review). It is defined as the duration of the final fixation toward a relevant target prior to the execution of the critical phase of movement and has been accepted as a measure of optimal psychomotor control. It is postulated that the $\mathrm{QE}$ allows for a period of cognitive preprogramming of movement parameters while minimizing distraction from other environmental or internal cues [20]. The critical movement for this specific task was considered as the arrival of the tool within "2 gaze cursors" distance (i.e., $2^{\circ}$ ) of the ball. The QE is therefore operationally defined as the final fixation on the ball prior to the arrival of the tool tip within $2^{\circ}$ of visual angle of the target.

Analysis

The first trial was considered a familiarization attempt for all participants, providing an insight into the testing protocol while limiting additional learning opportunities prior to testing. Data from the subsequent two trials were averaged to provide a mean value for each variable for each participant to be used for subsequent analyses. The researcher analysing the gaze data was experienced in performing such analyses and blind to the skill levels of the participants to protect against analysis bias.

Shapiro-Wilk tests revealed that all data were normally distributed. Differences between task completion time, total path length (TPL), total number of movements (TNoM), number of attempts required to grasp a ball, and quiet eye $(\mathrm{QE})$ variables for each group were analysed using a series of independent group $t$ tests. Differences in the locations (tools or target) fixated upon were subjected to a mixed-design $2 \times 2$ ANOVA (group $\times$ location), with Bonferroni-adjusted post hoc $t$ tests used to follow up significant interaction effects. All analyses were performed using SPSS 15.0 for Windows (SPSS Inc., Chicago, IL).

\section{Results}

Due to the corruption of a data storage device, performance and S-T data from the LAP Mentor for nine participants (2 experts and 7 novices) were lost. While the gaze and subcomponent task data are complete for all 25 participants, the analysis of the LAP Mentor data involves comparisons between eight novices and eight experienced operators. However, these reduced numbers still provide sufficient statistical power [19].

\section{Complete task: performance}

Experienced operators completed the task ( 9 balls) significantly more quickly than novices $\left(t_{14}=3.02, p<0.010\right.$; see Table 1).

\section{Complete task: tool movements ( $\mathrm{S}-\mathrm{T}$ interface)}

Experienced operators made significantly fewer total movements $\left(t_{14}=3.53, p<0.005\right)$ and had significantly shorter total tool path lengths $\left(t_{14}=3.40, p<0.005\right)$ than novices (see Table 1).

\section{Complete task: gaze strategy ( $\mathrm{S}-\mathrm{M}$ interface)}

The ANOVA on the percentage time spent fixating on each gaze location revealed a significant main effect for location $\left(F_{1,23}=27.2, p<0.001\right)$ and no significant main effect for

Table 1 Mean $( \pm$ SD) performance and S-T process measures for novice and experienced groups (from LAP Mentor)

\begin{tabular}{lll}
\hline Parameter & Novice & Experienced \\
\hline Completion time (s) & $203.8 \pm 76.5$ & $117.6 \pm 25.7$ \\
Total NoM & $265.7 \pm 97.9$ & $139.3 \pm 25.9$ \\
Total PL (cm) & $726.1 \pm 235.6$ & $438.9 \pm 38.0$
\end{tabular}

NoM number of movements, $P L$ path length 


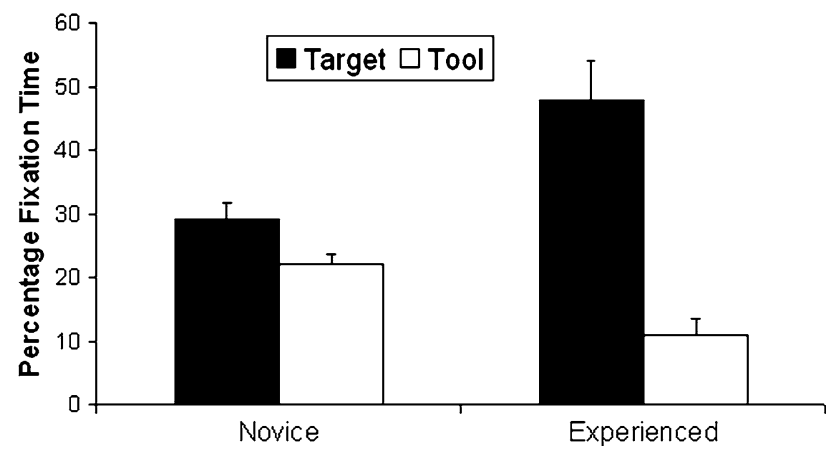

Fig. 3 The percentage of total fixation duration to target (jelly, ball, or endobag) and tool for novice and experienced surgeons ( \pm SEM)

Table 2 Mean $( \pm \mathrm{SD})$ quiet eye duration and number of ball grasp attempts during phase 2 of the task, for novice and experienced groups

\begin{tabular}{lcc}
\hline Parameter & Novice & Experienced \\
\hline Quiet eye (ms) & $597.3 \pm 194.6$ & $1123.4 \pm 539.1$ \\
Number of grasp attempts & $3.3 \pm 0.6$ & $2.5 \pm 0.7$ \\
\hline
\end{tabular}

ability level $\left(F_{1,23}=3.4, p=0.081\right)$. These results were qualified by a significant interaction effect $\left(F_{1,23}=13.2\right.$, $p<0.005)$. As Fig. 3 demonstrates, experts spent significantly more time fixating on the relevant target (jelly, ball, or endobag) than their novice counterparts $(p<0.005)$, while novices spent significantly more time tracking the tools than their expert counterparts $(p<0.005)$. Novices spent similar amounts of time fixating on the target ball and tracking the tools $(p=0.133)$, while experts spent significantly more time fixating the target balls compared to tool tracking $(p<0.001)$.

\section{Subcomponent task: eye-hand coordination}

Experienced operators had significantly longer quiet eye (QE) durations on the target ball $\left(t_{23}=3.49, p<0.005\right)$ and made significantly fewer grasp attempts $\left(t_{23}=2.92\right.$, $p<0.010$ ) than novice operators (Table 2).

\section{Discussion}

The aim of this research was to explore the coordination of eye and tool movements for a laparoscopic training task requiring coordinated and integrated hand movements. This study therefore extends recent research examining separate measures from the S-M (eye movement) and S-T (tool movement) interface for a more basic task [19]. The primary objective was to gain further insight into the strategies used by experienced and novice operators to overcome the perceptual constraints imposed by the laparoscopic environment. As the goal of simulated training is arguably the creation of a "pretrained novice," prepared for the operating room with reasonably automatic basic psychomotor and visual-spatial laparoscopic skills [6, 10], research examining indices related to these skills is clearly important.

The performance results are in accord with other recent studies and provide support for the discriminatory ability of the two-handed manoeuvres task [2, 12]: Experts were faster in completing the task and had more efficient tool movements than novices (Table 1). While it is often difficult to compare data between studies directly because of differences in procedures and measures reported [7], these results do add to the growing literature base supporting the evidence for validity of some of the LAP Mentor tasks [2, 10-14].

The feedback provided by the LAP Mentor software therefore allows the performance advantage of experts to be characterised and criterion scores for trainee performance to be set [2]. However, it is difficult to get an accurate conceptualisation of the perceptual processes underlying task completion without examining eye movements and indices related to eye-hand coordination. Our basic measure of gaze strategy (where the operators looked during task completion) revealed that experts generally used a target-focused gaze strategy during all three phases of the task, seldom needing to focus on the tool position. In comparison, novices adopted a switching strategy, focusing equally on both target and tool locations [19, 21] (Fig. 3). What does this reveal about differences in the perceptual strategies of both levels of operator?

Skilled psychomotor behaviour involves the ability to predict the consequences of one's actions and implement mapping rules relating motor and sensory signals [23]. Furthermore, eye movements and the gaze system that controls them play a key role in planning and controlling such precision motor actions [24]. Research examining skilful manual actions (e.g., pointing, grasping) has revealed that goal-directed hand movements are externally driven by target position and do not require visual feedback from the moving hand $[25,26]$. Peripheral vision appears to provide sufficient information to refine hand-movement control in this familiar and "natural" environment. This consistent finding can be summarised by the phrase "Keep your eyes on the prize!"

However, the laparoscopic environment is not natural, raising issues of depth perception, elongated tool use, the fulcrum effect, and limited degrees of freedom. Learning therefore involves the adaptation of previously acquired basic sensorimotor rules for manual reaching and grasping [27]. Previous research examining the learning of novel psychomotor tasks has demonstrated the important role of foveal vision and gaze shifts in learning novel mappings 
between hand motor commands and desirable sensory outcomes [23]. The results we have presented here for laparoscopic surgery reveal similar perceptual processes: Novices switch their attention between targets and tools as they develop the novel mappings required to successfully perform, whereas experienced operators no longer need such exploration and rely on the efficient, target-focused strategy used for less complex environments.

The results for the subcomponent of the task (picking up balls) revealed that it is not only the location of the fixation that is important, but also its timing. Approximately half of the experienced operators' fixations were not to the target (Fig. 3); however, they utilised a target-focused gaze when it was most needed (the quiet eye, QE). The experienced operators' QE periods were nearly twice that of the novice operators (Table 2). This steady gaze period appears to have helped guide these precision grasping movements, culminating in fewer unsuccessful attempts (Table 2). These results are therefore supportive of those examining proficiency differences in $\mathrm{QE}$ and performance in other motor tasks [22] and suggest that such measures of psychomotor control require further attention in the laparoscopic environment.

The implications of the current research findings extend beyond helping to clarify the processes underlying the skill advantage of expert laparoscopic operators, implying implicitly that these eye movement patterns must be learned and are made more efficient and effective through practice [23]. Given the importance of gaze information in planning and controlling psychomotor skills [24], research attention might profitably be applied to having novices replicate the gaze patterns and psychomotor control of experienced laparoscopic performers $[17,19]$. In the sports literature, for example, quiet eye training programmes have successfully expedited novice performance beyond that achieved by providing technical instructions related to movement parameters [28, 29].

We suggest that this advantage may be due to the benefits of learning the skills in an implicit manner, with little conscious awareness of how the skill is executed [30]. By focusing attention externally on relevant targets via gaze control rather than on the movements of the instruments, explicit rules are less likely to be accrued during learning. Masters et al. [31] previously demonstrated that by guiding novices with target information related to suture points, a suturing task could be learned in an implicit fashion. This may be important for subsequent operative performance as the accrual of explicit rules during learning is implicated in skill breakdown under conditions of stress, multitasking, and fatigue [31].

To conclude, the current study has further illuminated how surgeons utilize visual perceptual information to plan and control tool movements in a virtual reality laparoscopic environment. Performance results supported the evidence for validity of the two-handed manoeuvres task from the LAP Mentor basic skills training module. Results also supported previous findings from other domains that have revealed differences in the gaze strategies and psychomotor control (quiet eye) of learners and more experienced performers [20, 23]. Given recent calls for skills training programmes to be based on theoretical frameworks [2, 5, 8], future research should seek to test the utility of gaze training programmes to expedite basic surgical skill learning [17].

Acknowledgments This work was supported by a bilateral research grant from the Economic and Social Research Council, UK, and the Research Grants Council, Hong Kong (RES-000-22-3016), awarded to the first and last authors.

Disclosures Dr. Wilson, Dr. Brewer, Mr. Vine, Mr. McGrath, Mr. Defriend, and Professor Masters have no conflicts of interest or financial ties to disclose.

Open Access This article is distributed under the terms of the Creative Commons Attribution Noncommercial License which permits any noncommercial use, distribution, and reproduction in any medium, provided the original author(s) and source are credited.

\section{References}

1. Aggarwal R, Grantcharov TP, Eriksen JR, Blirup D, Kristiansen VB, Funch-Jensen P, Darzi A (2006) An evidence-based virtual reality training program for novice laparoscopic surgeons. Ann Surg 244:310-314

2. Aggarwal R, Crochet A, Dias A, Misra A, Ziprin P, Darzi A (2009) Development of a virtual reality training curriculum for laparoscopic cholecystectomy. Br J Surg 96:1086-1093

3. Gurusamy K, Aggarwal R, Palanivelu L, Davidson BR (2008) Systematic review of randomized controlled trials on the effectiveness of virtual reality training for laparoscopic surgery. Br J Surg 95:1088-1097

4. Sturm LP, Windsor JA, Cosman PH, Cregan P, Hewett PJ, Maddern GJ (2008) A systematic review of skills transfer after surgical simulation training. Ann Surg 248:166-179

5. Boehler ML, Schwind CJ, Rogers DA, Ketchum J, O'Sullivan E, Mayforth R, Quin J, Wohltman C, Johnson C, Williams RG, Dunnington $G$ (2007) A theory-based curriculum for enhancing surgical skillfulness. J Am Coll Surg 205:492-497

6. Gallagher AG, Ritter EM, Champion H, Higgins G, Fried MP, Moses G, Smith CD, Satava RM (2005) Virtual reality simulation for the operating room: proficiency-based training as a paradigm shift in surgical skills training. Ann Surg 241:364-372

7. Schout BMA, Hendrix AJM, Scheele F, Bemelmans BLH, Scherpbier AJJA (2010) Validation and implementation of surgical simulators: a critical review of present, past, and future. Surg Endosc 24:536-546

8. Verdaasdonk EG, Dankelman J, Lange JF, Stassen LP (2008) Incorporation of proficiency criteria for basic laparoscopic skills training: how does it work? Surg Endosc 22:2609-2615

9. Avodeji ID, Schijven M, Jakimowicz J, Greve JW (2007) Face validation of the Simbionix LAP Mentor virtual reality training module and its applicability in the surgical curriculum. Surg Endosc 21:1641-1649 
10. Andreatta PB, Woodrum DT, Birkmeyer JD, Yellamanchilli RK, Doherty GM, Gauger PG, Minter RM (2006) Laparoscopic skills are improved with LapMentor ${ }^{\mathrm{TM}}$ training: results of a randomized, double-blinded study. Ann Surg 243:854-863

11. Andreatta PB, Woodrum DT, Gauger PG, Minter RM (2008) LapMentor ${ }^{\mathrm{TM}}$ metrics possess limited construct validity. Simul Healthc 3:16-25

12. McDougall EM, Corica FA, Boker JR, Sala LG, Stoliar G, Borin JF, Chu FT, Clayman RV (2006) Construct validity testing of a laparoscopic surgical simulator. J Am Coll Surg 202:779-787

13. Yamaguchi S, Konishi K, Yasunaga T, Yoshida D, Kinjo N, Kobayashi K, Ieiri S, Okazaki K, Nakashima H, Tanoue K, Maehara Y, Hashizume M (2007) Construct validity for eye-hand coordination skill on a virtual reality laparoscopic surgical simulator. Surg Endosc 21:2253-2257

14. Zhang A, Hünerbein M, Dai Y, Schlag PM, Beller S (2008) Construct validity testing of a laparoscopic surgery simulator (LapMentor): evaluation of surgical skill with a virtual laparoscopic training simulator. Surg Endosc 22:1440-1444

15. Byrn JC, Schluender S, Divino CM, Conrad J, Gurland B, Shlasko E, Szold A (2007) Three-dimensional imaging improves surgical performance for both novice and experienced operators using the da Vinci Robot System. Am J Surg 193:519-522

16. Patel HR, Ribal MJ, Arya M, Nauth-Misir R, Joseph JV (2007) Is it worth revisiting laparoscopic three-dimensional visualization? A validated assessment. Urology 70:47-49

17. Wilson M, McGrath J, Coleman M (2010) Developing basic eyehand coordination skills for laparoscopic surgery using gaze training. BJU Int 105:1356-1358

18. Kocak E, Ober J, Berme N, Melvin WS (2005) Eye motion parameters correlate with level of experience in video-assisted surgery: objective testing of three tasks. J Laparoendosc Adv Surg Tech 15:575-580

19. Wilson M, McGrath J, Vine S, Brewer J, Defriend D, Masters R (2010) Psychomotor control in a virtual laparoscopic surgery training environment: gaze control parameters differentiate novices from experts. Surg Endosc 24:2458-2464
20. Vickers JN (1996) Visual control when aiming at a far target. J Exp Psychol Hum Percept Perform 2:324-354

21. Law B, Atkins MS, Kirkpatrick AE, Lomax AJ, Mackenzie CL (2004) Eye gaze patterns differentiate novice and experts in a virtual laparoscopic surgery training environment. In: Duchowski AT, Vertegaal R (eds) Proceedings of the eye tracking research \& application symposium, San Antonio, TX, March 22-24, 2004, pp 41-47

22. Vickers JN (2007) Perception, cognition and decision training: the quiet eye in action. Human Kinetics, Champaign, IL

23. Sailer U, Flanagan JR, Johansson RS (2005) Eye-hand coordination during learning of a novel visuomotor task. J Neurosci 25:8833-8842

24. Land MF (2009) Vision, eye movements, and natural behaviour. Vis Neurosci 26:51-62

25. Neggers SFW, Bekkering H (2000) Ocular gaze is anchored to the target of an ongoing pointing movement. J Neurophysiol 83:639-651

26. Sarlegna F, Blouin J, Bresciani JP, Bourdin C, Vercher JL, Gauthier GM (2003) Target and hand position information in the online control of goal-directed arm movements. Exp Brain Res 151:524-535

27. Holden JG, Flach JM, Donchin Y (1999) Perceptual-motor coordination in an endoscopic surgery simulation. Surg Endosc $13: 127-132$

28. Vine SJ, Wilson MR (2010) Quiet eye training: effects on learning and performance under pressure. J Appl Sport Psychol 22:361-376

29. Vine SJ, Wilson MR (2011) The influence of quiet eye training and pressure on attentional control in a visuo-motor task. Acta Psychol. doi:10.1016/j.actpsy.2010.12.008

30. Masters RSW (1992) Knowledge, (k)nerves and know-how: the role of explicit versus implicit knowledge in the breakdown of a complex motor skill under pressure. Br J Psychol 83:343-358

31. Masters RS, Lo CY, Maxwell JP, Patil NG (2008) Implicit motor learning in surgery: implications for multi-tasking. Surgery 143:140-145 